\title{
Fever and purpuric lesions in a preschool child
}

\author{
Meghan Bebbington $\mathrm{BSc}^{1}$, Joanne M Langley $\mathrm{MD}^{2,3,4}$, Conrad V Fernandez $\mathrm{MD}^{2,4}$
}

A three-year-old previously healthy girl presented to an emergency department with erythematous lesions on her nose, both cheeks and left calf associated with fever and irritability. Her guardian reported that the child's demeanour had changed from being pleasant and happy to general irritability approximately three weeks previously. Five days before presentation, she developed clear rhinorrhea and fever, but there was no cough, otalgia or headache. The child was reluctant to walk because of left leg pain. There was no history of travel or animal exposure. Her immunizations were current.

On examination she was febrile $\left(39.6^{\circ} \mathrm{C}\right)$, tachycardic (160 beats/min) and looked miserable. The chest was clear. A vibratory systolic ejection murmur was heard at the left sternal border without radiation and heart sounds were normal. There was no lymphadenopathy. The abdomen was soft and nontender with no organomegaly. A large, warm, tender, macular erythematous lesion was seen on the right face (Figure 1) with a central purplish bulla. The lesion on the tip of the nose consisted of a purplish-blue ulcer surrounded by erythema. A similar lesion was seen on the calf. The lesions were not indurated. The neck was supple.

The white blood cell count was $0.5 \times 10^{9} / \mathrm{L}$, with $3 \%$ neutrophils, $1 \%$ blast forms and $96 \%$ lymphocytes. The hemoglobin was $84 \mathrm{~g} / \mathrm{L}$ and the platelet count $87 \times 10^{9} / \mathrm{L}$. Blood cultures were done and empirical broad-spectrum antimicrobial therapy

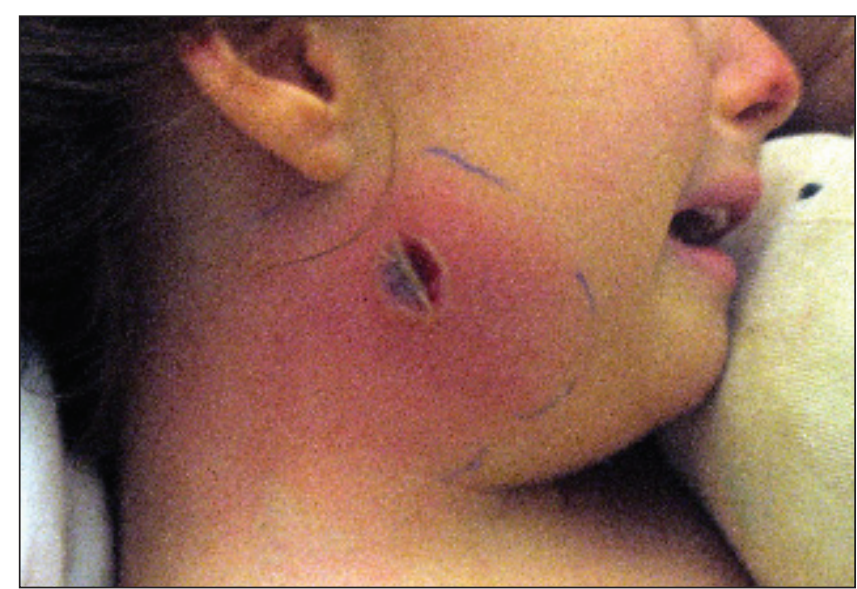

Figure 1) Lesions on the right side of the face and nose in a threeyear-old girl with irritability, fever and pancytopenia. A pen marking placed by the care team outlines the border of the erythema anteriorly

(ticarcillin/clavulanate, tobramycin and vancomycin) begun. A bone marrow biopsy and computed tomography scan of the neck were performed. The lesion on the right cheek, which opened spontaneously on day 2 , was swabbed and cultures were taken. What is the diagnosis?

\footnotetext{
${ }^{1}$ Faculty of Medicine; Departments of ${ }^{2}$ Pediatrics and ${ }^{3}$ Community Health and Epidemiology, Dalhousie University; ${ }^{4} I W K$ Health Centre, Halifax, Nova Scotia

Correspondence: Dr Joanne M Langley, IWK Health Centre, 5850 University Avenue, Halifax, Nova Scotia B3K 6 R8.

Telephone 902-470-8141,fax902-470-7232,e-mail jmlangle@dal.ca
} 


\section{DIAGNOSIS}

Bone marrow biopsy showed increased cellularity with a $44 \%$ population of blast cells consistent with precursor B cell acute lymphoblastic leukemia (ALL). A computed tomography scan of the neck showed significant soft tissue edema on the right side, with inflammation posterior to the mandible and extending down anterior to the right carotid artery. No evidence of abscess formation was present. Doppler ultrasonography of the neck showed no thrombus formation in the internal jugular vein.

The swab of the facial lesion showed growth of Staphylococcus aureus, sensitive to methicillin. Blood cultures were sterile. Based on the appearance of the lesions and the culture results, a clinical diagnosis of ecthyma gangrenosum (EG) was made. The child defervesced within a few days of the continuation of antibiotics (tobramycin, ticarcillin/clavulanate and cloxacillin). A two-week intravenous course of antibiotics was administered and the cancer treatment protocol begun at day 9 of presentation once the infection was clearly controlled.

\section{DISCUSSION}

ALL accounts for $75 \%$ of childhood leukemias, and may present with fever, fatigue, pallor or weight loss. Uncommonly, ALL is preceded by a preleukemic phase characterized by pancytopenia and bone marrow aplasia or hypoplasia $(1,2)$. Lifethreatening infections may develop in the context of overt neutropenia (as in this child) or inadequate bone marrow reserve, even with a normal absolute neutrophil count.

$E G$, the clinical diagnosis given to the present patient's skin lesions, is characterized by hemorrhagic pustules or necroticappearing lesions with surrounding erythema (3). EG is most commonly associated with Pseudomonas aeruginosa bacteremia in immunocompromised patients; however, numerous other organisms have been found to cause clinically identical lesions (4). A number of Gram-negative bacilli, herpes simplex virus, fungi (Aspergillus species, Candida albicans, Fusarium, Mucor and Rhizopus), and S aureus and Streptococcus pyogenes should also be included in the differential diagnosis because these have been reported as causes of EG-like lesions in the literature. The cutaneous lesions can result from direct inoculation or hematogenous spread and may occur anywhere in the body.

In the present case report, the culture grown from the patient was $S$ aureus, a common asymptomatic colonizer in healthy persons and a cause of cutaneous infection in the community including furuncles and impetigo, and systemic illnesses such as abscess, endocarditis, toxic shock syndrome and scalded skin syndrome (5). Although the blood culture in this patient was negative, hematogenous spread is the most likely explanation for the EG lesions on this child's leg and face. The site of the primary inoculum was not identified. EG lesions associated with $S$ aureus may also manifest as ecthyma with a gray-yellow crust surrounding a skin ulcer.

The present case demonstrates the importance of considering a wide range of pathogenic organisms as the etiological agent of EG-like cutaneous lesions and the need to initiate broad spectrum therapy. Fungal infection should also be considered (in particular, in the setting of bone marrow transplant or prolonged severe neutropenia) and appropriate diagnostic samples taken.

\section{REFERENCES}

1. Heegard ED, Madsen HO, Schmiegelow K. Transient pancytopenia preceding acute lymphoblastic leukaemia (pre-ALL) precipitated by parvovirus B19. Br J Haematol 2001;114:810-3.

2. Pouryousefi A, Foland J, Michie CA, Cummins M. Ecthyma gangrenosum as a very early herald of acute lymphoblastic leukaemia. J Paediatr Child H 1999;35:505-6.

3. Pouryousefi A, Foland J, Michie CA, Cummins M. Ecthymagangrenosum-like lesions associated with methicillin-resistant Staphylococcus aureus infection. J Paediatr Child Health 1999;35:505-6.

4. Reich HL, Fadeyl DW, Nalk NS, Honig PJ, Yan AC. Nonpseudomonal ecthyma gangrenosum. J Am Acad Dermatol 2004;50:114-7.

5. Howell ER, Phillips CM. Cutaneous manifestations of Staphylococcus aureus disease. Skinmed 2007;6:274-9. 


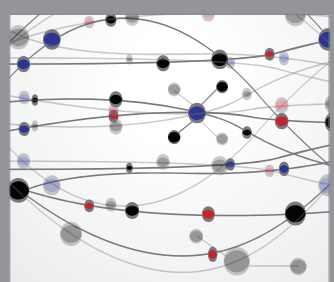

The Scientific World Journal


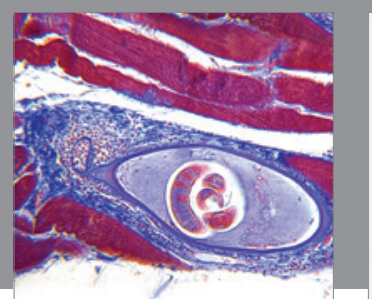

Gastroenterology Research and Practice

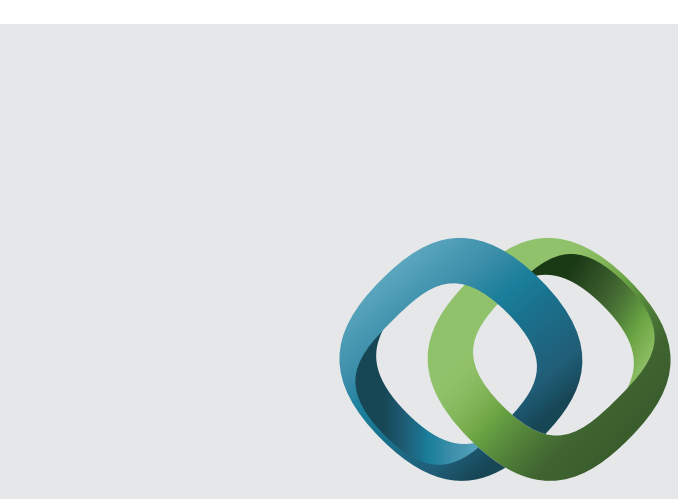

\section{Hindawi}

Submit your manuscripts at

http://www.hindawi.com
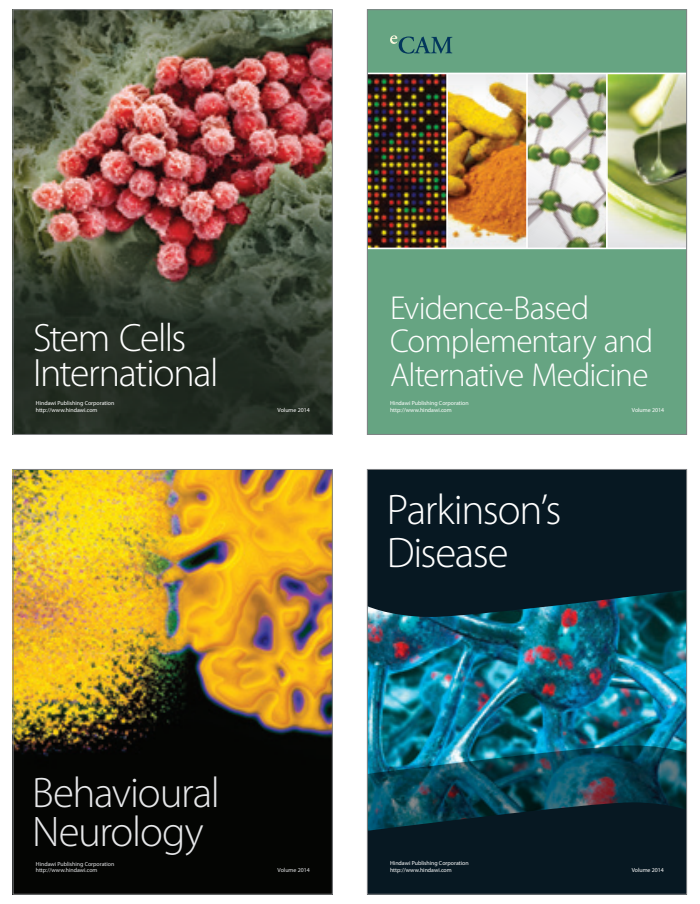


Disease Markers
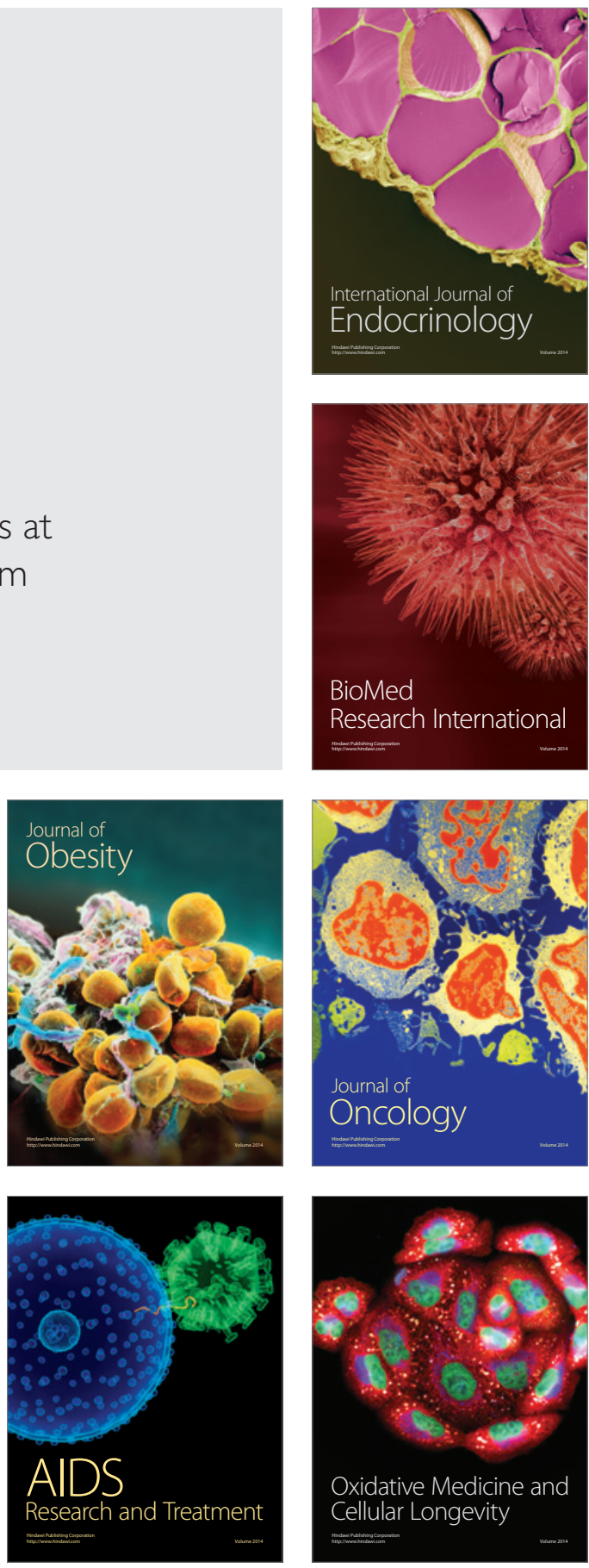